\title{
What to choose as radical local treatment for lung metastases from cob-rectal cancer: Surgery or radiofrequency ablation?
}

Citation for published version (APA):

Schlijper, R. C. J., Grutters, J. P. C., Houben, R., Dingemans, A-M. C., Wildberger, J. E., Van Raemdonck, D., Van Cutsem, E., Haustermans, K., Lammering, G., Lambin, P., \& De Ruysscher, D. (2014). What to choose as radical local treatment for lung metastases from cob-rectal cancer: Surgery or radiofrequency ablation? Cancer Treatment Reviews, 40(1), 60-67.

https://doi.org/10.1016/j.ctrv.2013.05.004

Document status and date:

Published: 01/02/2014

DOI:

10.1016/j.ctrv.2013.05.004

Document Version:

Publisher's PDF, also known as Version of record

Document license:

Taverne

Please check the document version of this publication:

- A submitted manuscript is the version of the article upon submission and before peer-review. There can be important differences between the submitted version and the official published version of record.

People interested in the research are advised to contact the author for the final version of the publication, or visit the DOI to the publisher's website.

- The final author version and the galley proof are versions of the publication after peer review.

- The final published version features the final layout of the paper including the volume, issue and page numbers.

Link to publication

\footnotetext{
General rights rights.

- You may freely distribute the URL identifying the publication in the public portal. please follow below link for the End User Agreement:

www.umlib.nl/taverne-license

Take down policy

If you believe that this document breaches copyright please contact us at:

repository@maastrichtuniversity.nl

providing details and we will investigate your claim.
}

Copyright and moral rights for the publications made accessible in the public portal are retained by the authors and/or other copyright owners and it is a condition of accessing publications that users recognise and abide by the legal requirements associated with these

- Users may download and print one copy of any publication from the public portal for the purpose of private study or research.

- You may not further distribute the material or use it for any profit-making activity or commercial gain

If the publication is distributed under the terms of Article $25 \mathrm{fa}$ of the Dutch Copyright Act, indicated by the "Taverne" license above, 
Anti-Tumour Treatment

\title{
What to choose as radical local treatment for lung metastases from colo-rectal cancer: Surgery or radiofrequency ablation?
}

\author{
Roel C.J. Schlijper a,c,*, Janneke P.C. Grutters ${ }^{b}$, Ruud Houben ${ }^{c}$, Anne-Marie C. Dingemans ${ }^{\mathrm{d}}$, \\ Joachim E. Wildberger ${ }^{\mathrm{e}}$, Dirk Van Raemdonck ${ }^{\mathrm{f}}$, Eric Van Cutsem ${ }^{\mathrm{g}}$, Karin Haustermans ${ }^{\mathrm{h}}$, \\ Guido Lammering ${ }^{\mathrm{c}}$, Philippe Lambin ${ }^{\mathrm{c}}$, Dirk De Ruysscher ${ }^{\mathrm{c}, \mathrm{h}}$
}

${ }^{a}$ Maastricht University, Faculty of Health, Medicine and Life Sciences, School of Medicine, Maastricht, The Netherlands

${ }^{\mathrm{b}}$ Department of Epidemiology, Biostatistics and HTA, Radboud University Nijmegen Medical Centre, The Netherlands

${ }^{\mathrm{c}}$ Department of Radiation Oncology (MAASTRO), GROW - School for Oncology and Developmental Biology, Maastricht University Medical Center, Maastricht, The Netherlands

${ }^{\mathrm{d}}$ Department of Pulmonology, GROW - School for Oncology and Developmental Biology, Maastricht University Medical Center, Maastricht, The Netherlands

${ }^{\mathrm{e}}$ Department of Radiology, Maastricht University Medical Center, Maastricht, The Netherlands

${ }^{\mathrm{f}}$ Department of Thoracic Surgery, University Hospital Leuven/KU Leuven, Leuven, Belgium

${ }^{g}$ Department of Digestive Oncology, University Hospital Leuven/KU Leuven, Leuven, Belgium

${ }^{\mathrm{h}}$ Department of Radiation Oncology, University Hospital Leuven/ KU Leuven, Leuven, Belgium

\section{A R T I C L E I N F O}

\section{Article history:}

Received 15 December 2012

Received in revised form 8 May 2013

Accepted 20 May 2013

\section{Keywords:}

Lung metastases

Colon cancer

Rectal cancer

Cancer

Surgery

RFA

\begin{abstract}
S U M M A R Y
Background: Long-term survival can be obtained with local treatment of lung metastases from colorectal cancer. However, it is unclear as to what the optimal local therapy is: surgery, radiofrequency ablation (RFA) or stereotactic radiotherapy (SBRT).

Methods: A systematic review included 27 studies matching with the a priori selection criteria, the most important being $\geqslant 50$ patients and a follow-up period of $\geqslant 24$ months. No SBRT studies were eligible. The review was therefore conducted on 4 RFA and 23 surgical series.

Results: Four of the surgical studies were prospective, all others were retrospective. No randomized trial was found. The reporting of data differed between the studies, which led to difficulties in the analyses. Treatment-related mortality rates for RFA and surgery were $0 \%$ and $1.4-2.4 \%$, respectively, whereas morbidity rates were reported inconsistently but seemed the lowest for surgery.

Conclusion: Due to the lack of phase III trials, no firm conclusions can be drawn, although most evidence supports surgery as the most effective treatment option. High-quality trials comparing currently used treatment modalities such as SBRT, RFA and surgery are needed to inform treatment decisions.
\end{abstract}

(C) 2013 Elsevier Ltd. All rights reserved.

\section{Introduction}

Colorectal cancer is the third most common tumor type in males, and the second in females, with in 2008 an incidence of 1.2 million new cases and a mortality rate of over 600.000 world-wide. ${ }^{1}$ In the Netherlands, approximately $20 \%$ of colorectal cancer patients have synchronic metastatic disease at time of diagnosis. ${ }^{2}$

Although the cure rate of patients with colorectal cancer has improved over the last decades, distant metastases are still of concern. Temporary remissions with systemic treatment can be obtained, but most of the time, cure remains elusive once distant

\footnotetext{
* Corresponding author. Address: Department of Radiation Oncology, Maastricht University Medical Centre, Maastricht, The Netherlands. Tel.: +31 884455 666; fax: +31884455667.
}

E-mail address: roel.schlijper@maastro.nl (R.C.J. Schlijper). metastases have occurred. In 1995, Hellman and colleagues proposed an intermediate state between localized cancer and distant metastases, called "oligo-metastases". ${ }^{3}$ In this concept, treatment of a few distant metastases with curative intent may still be possible, if all visible cancer can be eradicated with local treatments such as radiotherapy, surgery or radiofrequency ablation (RFA).

The observation that long-term survival may be achieved with surgical resection of liver metastases from colorectal cancer ${ }^{4}$ has been used as an argument supporting the oligo-metastases concept. Besides liver, also lung metastases from colorectal cancer have been treated with curative or radical intent. Most series deal with surgery, although RFA and Stereotactic Body Radiation Therapy or SBRT ${ }^{5}$ (also called SABR, Stereotactic Ablative RadioTherapy) are used as well. Most studies included patients with several types of primary tumors, whilst few studies reported outcomes on lung metastases from primary colorectal cancer, only. To the best of our knowledge, most series are retrospective or observational 
and no randomized series investigating survival outcome of these treatment modalities have been published. This was the reason for Treasure et al. ${ }^{6}$ to perform an ongoing phase III trial that investigates whether or not pulmonary metastasectomy in colorectal cancer influences survival.

Several systematic reviews regarding one or more of the three local treatment methods have been published. ${ }^{7-9}$ However, to the best of our knowledge, no systematic review has been reported comparing the outcome of surgery, RFA and SBRT specifically in the treatment of lung metastases of colorectal cancer. This was the aim of the current review.

\section{Methods}

\section{Search strategy and selection criteria}

The literature search was performed by using a broad strategy which was composed by following the PICO method ${ }^{10,11}$ (Supplementary Material 1). The complete search strategy is shown in Appendix 1 and was used to identify studies in Pub Med, EMBASE, Web of Science and the Cochrane Library from 2001 until the search date in October 2011.

For this review a priori selection criteria were established prior to the search and selection of articles. These included a minimal follow-up period of 24 months, a minimum of 50 patients included in the study with pulmonary metastases from colorectal cancer without constraint on previous therapies. Only original articles were included. Another limitation used was language, in which only English, German and Dutch articles were included. All inclusion- and exclusion criteria are summarized in Table 1.

In order to complete the search and to identify all relevant studies, the references of all eligible articles were manually searched for other potentially relevant studies.

\section{Outcomes}

One researcher conducted the search and selection of eligible studies. All articles were then evaluated by two independent reviewers. When available, the following data were obtained from the trials: patient and tumor characteristics, inclusion- and exclusion criteria, disease-free interval, treatment technique, follow-up, complications, tumor progression, recurrence rate, survival and prognostic factors.

One researcher (R.S.) reviewed all eligible studies, whereas the second extraction was performed by three reviewers (R.H., J.G. and D.D.R.). Data were extracted and tabulated independently in order to reach validity of the data (appendix 2 for extraction table). If outcomes differed, there was discussion between the reviewers until consensus was reached.

\section{Results}

\section{Search results}

The initial search in the four databases included 4727 articles in total, which were searched for duplicates using Endnote by which 453 duplicates were excluded.

Table 1

Inclusion and exclusion criteria for the literature search.

\begin{tabular}{ll} 
Follow-up period & $\geqslant 24$ months \\
Site of primary tumor & Colorectal carcinoma \\
Number of patients & $\geqslant n=50$ \\
Previous treatment & All therapies \\
Tumor stage & Stage IV \\
Type of metastasis & Recurrence or first secondary tumor \\
Study type & Reviews excluded \\
\hline
\end{tabular}

The remaining studies were manually evaluated and 226 more duplicates were excluded. Another 3250 studies were then excluded based on titles that were not relevant for this study (Fig. 1) leaving a total of 798 studies for further analysis.

Abstracts of all remaining 798 studies were then compared to the a priori selection criteria. Studies not matching these criteria were excluded as well. After this first selection, 27 relevant studies were identified and included in this review. Of these 27, the majority (23) focused on surgery, four examined RFA and none regarded SBRT.

\section{Description of the studies}

Of the twenty-seven studies that matched the selection criteria and were included in this review, four studies investigated RFA, all of which were retrospective. ${ }^{12-15}$ Three of these studies were performed in the same institute in Sydney, Australia ${ }^{13-15}$ with two of these papers ${ }^{13,14}$ investigating the same patient population. In our analysis, we used this population only once. All twenty-three remaining studies reviewed surgical metastasectomy, of which four prospective studies and the remaining retrospective studies. Study and patient characteristics are found in Table 2.

Despite the known importance according to the "oligo-metastases hypothesis", ${ }^{3}$ only six studies reported on the median or mean number of the pulmonary lesions. Yan et al. ${ }^{13,14}$ reported a median of 2 lesions per patient (range, 1-6), whilst Kanemitsu et al. ${ }^{16}$ and Pfannschmidt et al. ${ }^{17}$ found a median of 1 lesion per patient (ranges, 1-8 and 1-35 lesions, respectively). Two series only reported mean values of $2.3 \pm 2.1$ lesions $^{18}$ and $1.6 \pm 0.8$ lesions. $^{19}$

Other study and patient characteristics such as tumor diameter and follow-up durations are listed in Table 2.

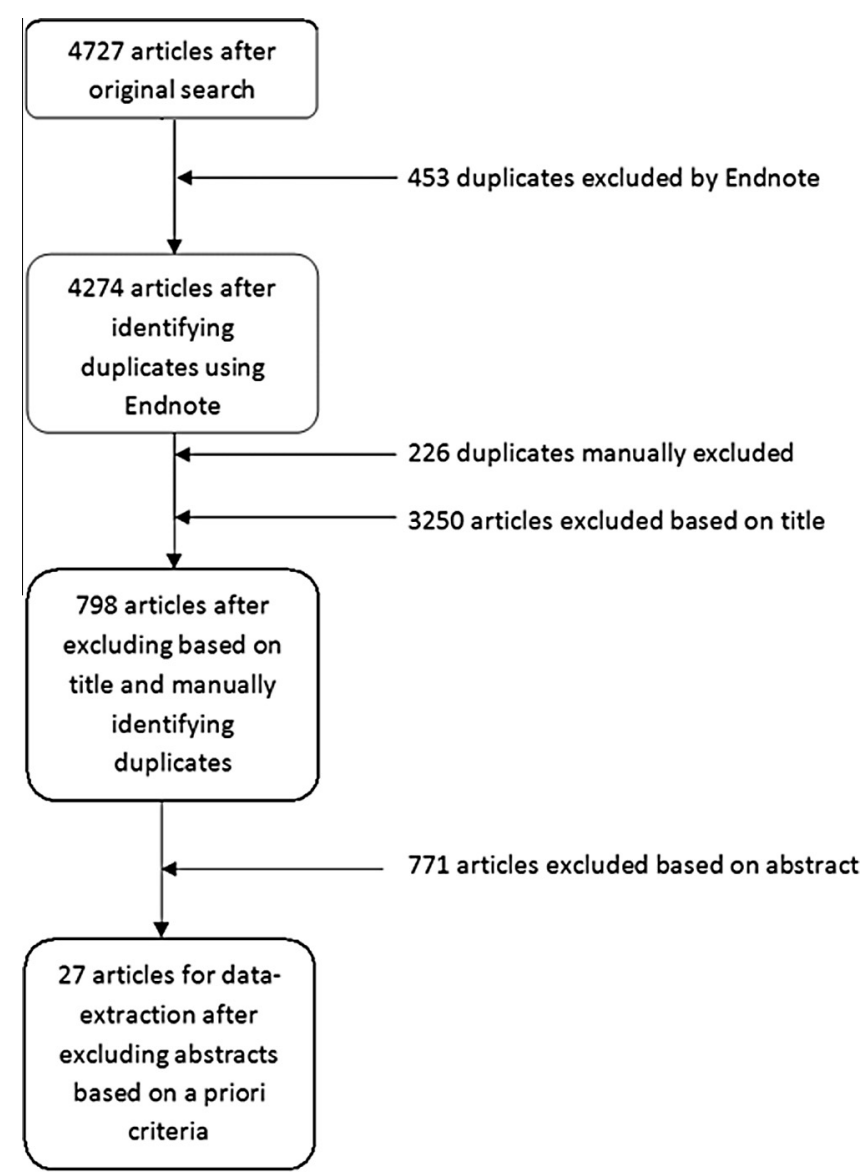

Fig. 1. Flowchart of selection process. 


\begin{tabular}{|c|c|c|c|c|c|}
\hline Study & Patient sex/age & No. of tumors & Involved lobes & Tumor diameter & Follow-up \\
\hline $\begin{array}{l}\text { Yamakado (2009); RFA, CT guided; } \\
\text { (Retrospective, } n=78 \text { ); Mie, } \\
\text { Japan }\end{array}$ & $\begin{array}{l}\text { Sex: M/F: } 53 / 25 ; \text { mean age } \\
66.1 \pm 9.8 \mathrm{y} \text { (range, } 40-87 \text { year); } \\
\leqslant 65 \text { year: } n=29 ;>65 \text { year: } n=49\end{array}$ & 1 lesion: $n=34 ; \geqslant 2$ lesions: $n=44$ & Unilateral $n=49$; Bilateral $n=29$ & $\begin{array}{l}\text { Mean max size: } 2.0 \pm 1.0 \mathrm{~cm} \text { (range } \\
0.6-6.0 \mathrm{~cm} \text { ); TD } \leqslant 3 \mathrm{~cm} n=70 \\
\text { TD }>3 \mathrm{~cm} n=8\end{array}$ & $\begin{array}{l}\text { Mean } 24.6 \text { months } \pm 17.6 \text { months } \\
\text { (range, } 6.0-84.1 \text { months) }\end{array}$ \\
\hline $\begin{array}{l}\text { Yan (2006); RFA, CT guided; } \\
\text { (Retrospective, } n=55 \text { ); Sydney, } \\
\text { Australia }\end{array}$ & $\begin{array}{l}\text { Sex: M/F: 33/22; mean age } \\
62 \pm 11 \text { year; } \leqslant 65 \text { year: } n=29 \\
>66 \text { year: } n=26\end{array}$ & $\begin{array}{l}1-2 \text { lesions: } n=39 ; 3-6 \text { lesions: } \\
n=16 ; \text { mean pp } 2 \text { lesions } \pm 2 \\
\text { median pp } 2 \text { lesions (range, } 1-6 \text { ) }\end{array}$ & 1 lobe: $n=39,2-4$ lobes $n=16$ & $\begin{array}{l}\text { Mean max size: } 2.1 \pm 1.1 \mathrm{~cm} ; \\
\mathrm{TD} \leqslant 3 n=42 ; \mathrm{TD}>3 \mathrm{~cm} n=13\end{array}$ & $\begin{array}{l}\text { Median } 24 \text { months (range, 6- } \\
40 \text { months): complete FU }\end{array}$ \\
\hline $\begin{array}{l}\text { Yan (2007); RFA, CT guided; } \\
\text { (Retrospective, } n=55 \text { ); Sydney, } \\
\text { Australia }\end{array}$ & $\begin{array}{l}\text { Sex: M/F: } 33 / 22 ; \text { mean age } \\
62 \pm 11 \text { year; } \leqslant 65 \text { year: } n=29 \\
>65 \text { year: } n=26\end{array}$ & $\begin{array}{l}1-2 \text { lesions: } n=39 ; 3-6 \text { lesions: } \\
n=16 ; \text { mean pp } 2 \text { lesions } \pm 2 ; \\
\text { median pp } 2 \text { lesions (range, } 1-6 \text { ) }\end{array}$ & 1 lobe: $n=39,2-4$ lobes $n=16$ & $\begin{array}{l}\text { Mean max size: } 2.1 \pm 1.1 \mathrm{~cm} ; \\
\mathrm{TD} \leqslant 3 n=42 ; \mathrm{TD}>3 \mathrm{~cm} n=13\end{array}$ & $\begin{array}{l}\text { Median } 24 \text { months (range, } 6- \\
40 \text { months): complete FU }\end{array}$ \\
\hline $\begin{array}{l}\text { Chua (2010); RFA, CT guided; } \\
\text { (Retrospective, } n=108) \text {; Sydney, } \\
\text { Australia }\end{array}$ & $\begin{array}{l}\text { Sex: M/F: } 83 / 65 ; \text { median age } \\
63 \text { year (range } 30-85 \text { ); } \leqslant 60 \text { year: } \\
n=50 ;>60 \text { year: } n=98\end{array}$ & $\begin{array}{l}1-2 \text { lesions: } n=104 ; \geqslant 3 \text { lesions: } \\
n=44\end{array}$ & Unilateral $n=107$; Bilateral $n=41$ & $\begin{array}{l}\text { Mean max size: } 2 \pm 2 \mathrm{~cm} \\
\mathrm{TD} \leqslant 4 \mathrm{~cm} n=89 ; \mathrm{TD}>4 \mathrm{~cm} n=59\end{array}$ & $\begin{array}{l}\text { Median } 29 \text { months (range, } 2- \\
103 \text { months) }\end{array}$ \\
\hline $\begin{array}{l}\text { Brouquet (2011); Surgery; (Retro- / } \\
\text { prospective, } n=112) \text {; Houston, } \\
\text { U.S. }\end{array}$ & $\begin{array}{l}\text { Sex: } \mathrm{M} / \mathrm{F}: 78 / 34 ; \text { mean age } \\
55 \text { year } \pm 11 \text { year }\end{array}$ & Mean pp 2.3 lesions \pm 2.1 & Unilateral $n=78$; Bilateral $n=34$ & Mean size $1.5 \mathrm{~cm} \pm 1.2$ & $\begin{array}{l}\text { Median } 49 \text { months (range, } 0.1- \\
159.0 \text { months) }\end{array}$ \\
\hline $\begin{array}{l}\text { Koga (2006); Surgery; } \\
\quad \text { (Retrospective, } n=58 \text { ); Tokyo, } \\
\text { Japan }\end{array}$ & $\begin{array}{l}\text { Sex: M/F: } 30 / 28 ; \text { median age } \\
63 \text { year (range, 33-87 year) }\end{array}$ & $\begin{array}{l}1 \text { lesion } n=23 ; 2 \text { lesions } n=16 ; 3 \\
\text { lesions } n=10 ; 4-12 \text { lesions } n=9\end{array}$ & Unilateral $n=45$; Bilateral $n=13$ & $\mathrm{TD} \leqslant 3 \mathrm{~cm} n=31 ; \mathrm{TD}>3 \mathrm{~cm} n=27$ & $\begin{array}{l}\text { Median } 24 \text { months (range, 5- } \\
233 \text { months) }\end{array}$ \\
\hline $\begin{array}{l}\text { Ogata (2005); Surgery; } \\
\quad \text { (Retrospective, } n=76) \text {; Fukuoka, } \\
\text { Japan }\end{array}$ & $\begin{array}{l}\text { Sex: M/F: 39/37; mean age } \\
62.9 \text { year (range, } 41-83 \text { year) }\end{array}$ & $\begin{array}{l}1 \text { lesion } n=56 ; 2 \text { lesions } n=15 ; 3 \\
\text { lesions } n=4 ; 4 \text { lesions } n=1\end{array}$ & Unilateral $n=69 ;$ Bilateral $n=7$ & $\begin{array}{l}\text { Mean size } 2.69 \pm 1.47 \text { (range, } 0.7- \\
10.0 \mathrm{~cm} \text { ); TD }<3 \mathrm{~cm} n=29 \\
\mathrm{TD} \geqslant 3 \mathrm{~cm} n=47\end{array}$ & $\begin{array}{l}\text { Median } 47 \text { months (range, } 2- \\
204 \text { months) }\end{array}$ \\
\hline $\begin{array}{l}\text { Kanemitsu (2010); Surgery; } \\
\text { (Prospective); Nagoya, Japan }\end{array}$ & $\begin{array}{l}\text { Sex: M/F: } 27 / 31 ; \text { median age } \\
62 \text { year (range, } 36-84 \text { year) }\end{array}$ & Median 1 lesion (range, 1-8) & Unilateral $n=45$; Bilateral $n=13$ & $\begin{array}{l}\text { Median max size } 2.0 \mathrm{~cm} \text { (range 1- } \\
8 \mathrm{~cm} \text { ) }\end{array}$ & $\begin{array}{l}\text { Median: } 39 \text { months (range, } 5- \\
94 \text { months) (total group); median } \\
51 \text { months (range, 29-89 months) } \\
\text { (surviving patients) }\end{array}$ \\
\hline $\begin{array}{l}\text { Park (2010); Surgery; } \\
\quad \text { (Retrospective, } n=195 \text { ); Seoul, } \\
\quad \text { South Korea }\end{array}$ & $\begin{array}{l}\text { Sex: } \mathrm{M} / \mathrm{F}: 122 / 73 ; \text { median age } \\
58 \text { year } \pm 9.5 \text { year }\end{array}$ & $\begin{array}{l}1 \text { lesion } n=112 ; 2-4 \text { lesions } \mathrm{I}=70 \\
>5 \text { lesions } n=10 \text {; unknown } n=3\end{array}$ & Unilateral $n=146$; Bilateral $n=48$ & $\begin{array}{l}\text { Max size: } \leqslant 4 \mathrm{~cm} n=170 ;>4 \mathrm{~cm} \\
n=17 ; \text { undocumented } n=8\end{array}$ & $\begin{array}{l}\text { Median } 42.3 \text { months; loss-to } \\
\text { follow-up } n=1\end{array}$ \\
\hline $\begin{array}{l}\text { Headrick (2001); Surgery; } \\
\quad \text { (Retrospective, } n=58 \text { ); } \\
\text { Rochester, U.S. }\end{array}$ & $\begin{array}{l}\text { Sex: M/F: } 37 / 21 ; \text { median age } \\
59 \text { year (range, } 31-82 \text { year) }\end{array}$ & $\begin{array}{l}1 \text { lesions } n=31 ; 2 \text { lesions } n=13 ; 3 \\
\text { lesions } n=10 ; 4 \text { lesions } n=4\end{array}$ & $\mathrm{~N} / \mathrm{A}$ & $\mathrm{N} / \mathrm{A}$ & $\begin{array}{l}\text { Median } 62 \text { months (range, } 6- \\
201 \text { months) }\end{array}$ \\
\hline $\begin{array}{l}\text { Hornbech (2011); Surgery; } \\
\quad \text { (Retrospective, } n=53 \text { ); } \\
\text { Copenhagen, Denmark }\end{array}$ & $\mathrm{N} / \mathrm{A}$ & & Unilateral $n=41$; Bilateral $n=12$ & $\mathrm{~N} / \mathrm{A}$ & $\begin{array}{l}\text { Mean for all tumor sites; } \\
61.6 \text { months. Only analysis with } \\
\text { patients with minimal FU of } \\
36 \text { months }\end{array}$ \\
\hline $\begin{array}{l}\text { Inoue (2004); Surgery; } \\
\quad \text { (Retrospective, } n=128 \text { ); Osaka, } \\
\text { Japan }\end{array}$ & $\begin{array}{l}\text { Sex: M/F: } 85 / 43 ; \text { mean age } \\
61.8 \text { year (range, 39-78 year) }\end{array}$ & 1 lesion $n=95 ; \geqslant 2$ lesions $n=33$ & $\mathrm{~N} / \mathrm{A}$ & $\mathrm{N} / \mathrm{A}$ & $\begin{array}{l}\text { Mean } 85.9 \text { months; loss-to follow- } \\
\text { up } n=7\end{array}$ \\
\hline
\end{tabular}
Japan

Chen (2009); Surgery Japan

Hwang (2010); Surgery; (Prospective, $n=125$ ); Gyeonggi-do, South Korea

Riquet (2010); Surgery;

$\begin{array}{ll}\text { Retrospective, } n=117) ; \text { Paris, } & \text { Sex: M/F: 74/53; median age } \\ \text { Rear (range, } 36-85 \text { year); }\end{array}$ France

Rama (2008); Surgery: $\begin{array}{ll}\text { Sex: M/F: 42/19; mean age } \\ \text { (Retrospective } n=62) \text {. Lisbon, } & 61 \text { year + } 14 \text { year (range, 30- }\end{array}$ $n=62$ ); Lisbon, 61 year \pm 14 year (range, 30 Portugal

Takakura (2009); Surgery; (Retrospective, $n=56$ ); Hiroshima, Japan

Lin (2009); Surgery; (Prospective, $n=63)$; Taipei, Taiwan

Sex: M/F: 54/30; median age
65 year (range, $41-86$ year)

Sex: M/F: 75/50; median age 60 year (range, $32-80$ year); $\leqslant 60$ $=64 ;>60 n=61$ sear $n=62 ;>65$ year $n=65$ 80 year)

Sex: M/F: 26/30; median age 64 year (range $42-76$ year)

Sex: M/F: 39/24; median age 58.7 year (range $32-$
1 lesion $n=22$; 2-4 lesions $n=51$; Unilateral $n=68$; Bilateral $n=16$ $\geqslant 5$ lesions $n=11$

1 lesion $n=77$; $\leqslant 3$ lesions $n=109$; Unilateral $n=94$; Bilateral $n=31$ $>3$ lesions $n=16$

Total 314; 1 lesion $n=75 ; \geqslant 2 \quad \mathrm{~N} / \mathrm{A}$

lesions $n=42$

Mean 1.6 lesions \pm 0.8 ; 1 lesion: $\quad$ Unilateral $n=59$; Bilateral $n=2$ $n=37 ; 2$ lesions $n=13 ; \geqslant 3$ lesions

$n=11$

1 lesion $n=37$; $\geqslant 2$ lesions $n=19 \quad$ Unilateral $n=44$; Bilateral $n=12$ (range 2-14)

1 lesion $n=41 ; 2$ lesions $n=14 ; 3 \quad$ Unilateral $n=51$; Bilateral $n=12$ lesions $n=5 ; \geqslant 4$ lesions $n=3$
$\mathrm{TD}<3 \mathrm{~cm} \mathrm{n}=18 ; \mathrm{TD} \geqslant 3 \mathrm{~cm} n=66$ Median 28 months (range, 3135 months)

Median size $1.8 \mathrm{~cm}$ (range, $0.5-$ $7.0 \mathrm{~cm}) ; \mathrm{TD} \leqslant 2 \mathrm{~cm} n=85$;

$\mathrm{TD}>2 \mathrm{~cm} n=40$

Median size $3.0 \pm 1.9 \mathrm{~cm}$ (range, Median 46 months (range, 2$0.2-9.0 \mathrm{~cm}) ; \mathrm{TD}<2.9 \mathrm{~cm} n=64$ TD $>3.0 \mathrm{~cm} n=53$

$\mathrm{TD} \leqslant 3 \mathrm{~cm} n=44 ; \mathrm{TD}>3 \mathrm{~cm} n=17 \quad$ Mean 39 months \pm 39 months (range, 4-173 months); complete $n=56, n=5$ excluded from analysis Mean 30 months

Median
$8.8 \mathrm{~cm})$

Mean size $2.77 \mathrm{~cm}$ (range 0.5 $8.6 \mathrm{~cm}$ ); TD < $3 \mathrm{~cm} n=40$;
Median 37.3 months (range, 12 122 months) 
Table 2 (continued)

\begin{tabular}{|c|c|c|c|c|c|}
\hline Study & Patient sex/age & No. of tumors & Involved lobes & Tumor diameter & Follow-up \\
\hline $\begin{array}{l}\text { Zabaleta (2011); Surgery; } \\
\quad \text { (Retrospective, } n=84 \text { ); San } \\
\text { Sebastian, Spain }\end{array}$ & $\begin{array}{l}78 \text { year)>60 year } n=29 \\
\text { Sex: M/F: } 60 / 24 ; \text { median age } \\
65.5 \text { year; mean age } 65.4 \text { year }\end{array}$ & 1 lesion $n=65 ; \geqslant 2$ lesions $n=19$ & $\mathrm{~N} / \mathrm{A}$ & $\begin{array}{l}\mathrm{TD} \geqslant 3 \mathrm{~cm} \mathrm{n}=23 \\
\mathrm{TD}>2 \mathrm{~cm} n=48 ; \mathrm{TD}<2 \mathrm{~cm} n=36\end{array}$ & $\begin{array}{l}\text { Median } 43 \text { months (range, } 0- \\
130 \text { months) }\end{array}$ \\
\hline $\begin{array}{l}\text { Pfannschmidt (2003); Surgery; } \\
\text { (Retrospective, } n=167 \text { ); } \\
\text { Heidelberg, Germany }\end{array}$ & $\begin{array}{l}\text { Sex: M/F: } 103 / 64 ; \text { mean age } \\
60.2 \text { year (range, } 25-81 \text { year) }\end{array}$ & $\begin{array}{l}\text { Median } 1 \text { lesion (range, } 1-35 \text { ); } 1 \\
\text { lesion } n=84 ; \geqslant 2 \text { lesions } n=83\end{array}$ & $\mathrm{~N} / \mathrm{A}$ & $\mathrm{N} / \mathrm{A}$ & $\begin{array}{l}\text { Mean } 58.6 \pm 42.7 \text { months (range, } \\
0.5-183.9 \text { months) }\end{array}$ \\
\hline $\begin{array}{l}\text { Higashiyama (2003); Surgery; } \\
\text { (Retrospective, } n=100) \text {; Osaka, } \\
\text { Japan }\end{array}$ & $\begin{array}{l}\text { Sex: M/F: } 61 / 39 ; \text { mean age } \\
60.3 \text { year (range, } 39-79 \text { year); }<61 \\
n=49 ; \geqslant 61 n=51\end{array}$ & $\begin{array}{l}\text { On radiography: } 1 \text { lesion } n=49 ; 2 \\
\text { lesions } n=25 ; 3 \text { lesions } n=18 ; 4 \\
\text { lesions } n=4 ; 5 \text { lesions } n=1 ; 6 \\
\text { lesions } n=3 \text { : Perioperative: } 1 \\
\text { lesion } n=55 ; 2 \text { lesions } n=23 ; 3 \\
\text { lesions } n=14 ; 4 \text { lesions } n=3 ; 5 \\
\text { lesions } n=2 ; 6 \text { lesions } n=3\end{array}$ & Unilateral $n=79$; Bilateral $n=21$ & $\begin{array}{l}\text { Max size } 0.2-11.0 \mathrm{~cm} ; \mathrm{TD} \leqslant 3 \\
n=59 ; \mathrm{TD}>3 n=41\end{array}$ & $\begin{array}{l}\text { Median } 30.3 \text { months (range, 3.6- } \\
168.7 \text { months) }\end{array}$ \\
\hline $\begin{array}{l}\text { Nakajima (2007); Surgery; } \\
\quad \text { (Retrospective, } n=143 \text { ); Tokyo, } \\
\text { Japan }\end{array}$ & $\begin{array}{l}\text { Open: sex: } \mathrm{M} / \mathrm{F}: 49 / 22 \text {; age } \\
59.8 \text { year } \pm 9.9 ; \text { Thoracoscopy: } \mathrm{M} / \mathrm{F} \text { : } \\
43 / 29 \text {; age } 63.3 \text { year } \pm 11.0\end{array}$ & $\begin{array}{l}\text { Open } 3.4 \text { lesions } \pm 4.5 \text {; scopy } 1.6 \\
\text { lesions } \pm 0.9\end{array}$ & $\mathrm{~N} / \mathrm{A}$ & $\begin{array}{l}\text { Max TD: open } 2.7 \mathrm{~cm} \pm 1.8 \text {; scopy } \\
1.5 \mathrm{~cm} \pm 0.9\end{array}$ & $\begin{array}{l}46.7 \text { months } \pm 54.3 \text { months (open } \\
\text { group); } 34.4 \text { months } \pm 22.0 \text { months } \\
\text { (thoracoscopy group) }\end{array}$ \\
\hline $\begin{array}{l}\text { Shiono (2004); Surgery; ( . . - } \\
\text { spective, } n=87 \text { ); Tokyo, Japan }\end{array}$ & $\begin{array}{l}\text { Sex M/F: } 57 / 30 \text {; median age } 61 \text { year } \\
\text { (range, 23-83 year) }\end{array}$ & $\begin{array}{l}1 \text { lesion } n=66 ; 2 \text { lesions } n=9 ; 3 \\
\text { lesions } n=8 ; 4 \text { lesions } n=3 ; 5 \\
\text { lesions } n=1\end{array}$ & $\mathrm{~N} / \mathrm{A}$ & $\begin{array}{l}\text { Median size } 1.8 \mathrm{~cm} \text { (range 0.7- } \\
6.7 \mathrm{~cm} \text { ); TD } \leqslant 3 \mathrm{~cm} n=66 \\
\text { TD > } 3 \mathrm{~cm} n=21\end{array}$ & $\begin{array}{l}\text { Median } 32 \text { months (range, } 1- \\
110 \text { months) }\end{array}$ \\
\hline $\begin{array}{l}\text { Sakamoto (2010); Surgery; } \\
\quad \text { (Retrospective, } n=59 \text { ); Tokyo, } \\
\text { Japan }\end{array}$ & $\begin{array}{l}\text { Total group: M/F: } 162 / 81 \text {, median } \\
\text { age } 66 \text { year (range, 9-86 year); } \\
\text { Metastasis group M/F: } 81 / 52 \text {, } \\
\text { median age } 63 \text { year (range } 9- \\
82 \text { year) }\end{array}$ & $\mathrm{N} / \mathrm{A}$ & $\mathrm{N} / \mathrm{A}$ & $\begin{array}{l}\text { Total group: median size } 1.5 \mathrm{~cm} \\
\text { (range } 0.2-9.5 \mathrm{~cm} \text { ); metastasis } \\
\text { group median size } 1.5 \mathrm{~cm} \text { (range } \\
0.2-7.5 \mathrm{~cm} \text { ); Metastasis group } \\
\text { TD }<3 \mathrm{~cm} n=117 ; \text { TD } \geqslant 3 \mathrm{~cm} \\
n=16\end{array}$ & $\begin{array}{l}\text { Median: } 37 \text { months (range, } 0- \\
132 \text { months) (for both total group } \\
\text { and metastasis group) }\end{array}$ \\
\hline $\begin{array}{l}\text { Kanzaki (2011); Surgery; } \\
\quad \text { (Retrospective, } n=156 \text { ); Osaka, } \\
\text { Japan }\end{array}$ & $\begin{array}{l}\text { Sex: M/F: } 91 / 65 \text {; mean age } 62 \text { year } \\
\text { (range, 39-83 year) }\end{array}$ & $\begin{array}{l}1 \text { lesion } n=100 ; 2 \text { lesions } n=32 \\
\geqslant 3 \text { lesions } n=24\end{array}$ & Unilateral $n=130$; Bilateral $n=26$ & $\begin{array}{l}\operatorname{Max} \mathrm{TD} \leqslant 3 \mathrm{~cm} n=115 ; \mathrm{TD}>3 \mathrm{~cm} \\
n=41\end{array}$ & $\begin{array}{l}\text { Median } 43 \text { months (range, } 4- \\
270 \text { months) }\end{array}$ \\
\hline $\begin{array}{l}\text { Rolle (2006); Surgery; ( . ..-spective, } \\
\quad n=91 \text { ); Dresden, Germany }\end{array}$ & $\begin{array}{l}\text { Sex: M/F: } 164 / 164 ; \text { mean age } \\
61 \text { year (range, } 20-80 \text { year) }\end{array}$ & $\begin{array}{l}\text { Total } 3267 \text { nodules (average } 10 / p t) \\
1 \text { lesion } n=92 ; \geqslant 4 \text { lesions } n=161 \\
\geqslant 10 \text { lesions } n=69 ; \geqslant 20 \text { lesions } \\
n=30\end{array}$ & Unilateral $n=165$; Bilateral $n=113$ & Range of TD $0.3-8 \mathrm{~cm}$ & $\mathrm{~N} / \mathrm{A}$ \\
\hline $\begin{array}{l}\text { Rolle (2006); Surgery; ( . . -spective, } \\
\quad n \text { = 91); Dresden, Germany }\end{array}$ & $\begin{array}{l}\text { Sex: } M / F: 164 / 164 ; \text { mean age } \\
61 \text { year (range, } 20-80 \text { year) }\end{array}$ & $\begin{array}{l}\text { Total } 3267 \text { nodules (average } 10 / p t \text { ); } \\
1 \text { lesion } n=92 ; \geqslant 4 \text { lesions } n=161 \\
\geqslant 10 \text { lesions } n=69 ; \geqslant 20 \text { lesions } \\
n=30\end{array}$ & Unilateral $n=177$; Bilateral $n=151$ & $\mathrm{~N} / \mathrm{A}$ & $\begin{array}{l}\text { Mean } 31 \text { months, median } \\
22.5 \text { months (range, } 1- \\
198 \text { months) }\end{array}$ \\
\hline
\end{tabular}

$\mathrm{pp}=$ per patient

$=$ Tumor Diameter. 
Local control

All treatment outcome data are summarized in Table 3.

Local control of treated metastases differed between the studies. Data on local control were not always provided but if so, these data were reported in either progression free survival (PFS) or disease free survival (DFS). Median PFS reported in the RFA studies by Yan et al. ${ }^{13,14}$ was 15 months (range, 3-40), while 2-year local PFS was $56 \%{ }^{14}$ Data on local control were not provided in other RFA studies.

Six surgery studies reported local control. Brouquet et al. ${ }^{18}$ and Ogata et al. ${ }^{20}$ provided DFS data for patients treated with both hepatic and pulmonary metastasectomy, with 5-year DFS being $25 \%$ and $27 \%$ respectively. Ogata et al. ${ }^{20}$ also reported on data on 5 -year lung DFS being 35\%.

The 5-year DFS reported by Chen et al. ${ }^{21}$ and Lin et al. ${ }^{22}$ were $34.4 \%$ and $19.5 \%$, respectively. Rama et al. ${ }^{19}$, reported a mean DFS of 52 months \pm 6 months and Takakura et al. ${ }^{23}$ found a DFS of 13 months (range, 2-35 months).

\section{Overall survival}

Median Overall Survival (OS) was reported in 11 of 27 articles. For RFA, Yamakado et al. ${ }^{12}$ reported a median OS of 38 months.
The two articles by Yan et al. ${ }^{13,14}$ reported from the one dataset of 55 patients and provided a median OS of 33 months (range, 440 months). The fourth study concerning RFA by Chua et al. ${ }^{15}$ reported a median OS of 51 months ( $95 \% \mathrm{CI}, 19-83$ months) for the total study population of 148 patients with pulmonary metastases from several primary tumors. Outcome data for the colorectal cancer patient subgroup were not available.

Regarding surgery, in the study of Brouquet et al. ${ }^{18}$, the median OS was 58 months for patients with both liver and lung metastasectomy. Retrospective studies by Koga et al. ${ }^{24}$ and Hornbech et al. ${ }^{25}$ reported median OS of 27 months and 33.5 months, respectively. Hornbech et al. ${ }^{25}$ also analyzed the median survival of subgroups with (15.4 months) or without (28.6 months) concurrent hepatic metastases. Hwang et al. $^{26}$ conducted a prospective study with a median OS of 37 months whereas the retrospective studies by Zabaleta et al. ${ }^{27}$ and Pfannschmidt et al. ${ }^{17}$ reported median OS of 72 months (range, 0-129 months) and 40.2 months, respectively.

One study (Rama et al. $^{19}$ ) reported only a mean OS of 67 months \pm 16 months.

\section{Survival rates}

Good comparison between survival rates was difficult since these outcome data were differently reported between the papers.

Table 3

Treatment outcome.

\begin{tabular}{|c|c|c|c|}
\hline Study & $\begin{array}{l}\text { Number of } \\
\text { patients }(\mathrm{n})\end{array}$ & $\begin{array}{l}\text { Retro- }(\mathrm{R}) \text { or } \\
\text { prospective }(\mathrm{P})\end{array}$ & Treatment outcome \\
\hline $\begin{array}{c}\text { Yamakado } \\
\text { (2009) }\end{array}$ & 78 & $\mathrm{R}$ & SR 1-year/3-year/5-year: 83.9\%/56.1\%/34.9\%; Median Survival 38.0 months \\
\hline Yan (2006) & 55 & $\mathrm{R}$ & $\begin{array}{l}\text { PFS median } 15 \text { months (range, 3-40 months); SR 1-year/2-year/3-year: 85\%/64\%/46\%; Median OS } 33 \text { months } \\
\text { (range, } 4-40 \text { months) }\end{array}$ \\
\hline Yan (2007) & 55 & $\mathrm{R}$ & $\begin{array}{l}\text { PFS median } 15 \text { months (range, 3-40 months); Local PFS 1-year/2-year: 74\%/56\%; SR 1-year/2-year/3-year: } \\
\text { 85\%/64\%/46\%; Median OS } 33 \text { months (range, 4-40 months) }\end{array}$ \\
\hline Chua (2010) & 108 & $\mathrm{R}$ & $\begin{array}{l}\text { PFS median } 11 \text { months (95\% CI, 9-14 months); SR 3-year/5-year: 60\%/45\%; median OS } 51 \text { months (95\% CI, 19- } \\
83 \text { months) }\end{array}$ \\
\hline $\begin{array}{l}\text { Brouquet } \\
\text { (2011) }\end{array}$ & 112 & $\mathrm{P}$ & $\begin{array}{l}\text { DFS 3-year/5-year: } 28 \% / 25 \% \text { (both liver and lung metastasis) SR 3-year/5-year: } 71 \% / 50 \% \text {; median } 58 \text { months } \\
\text { (after liver + lung resection }\end{array}$ \\
\hline Koga (2006) & 58 & $\mathrm{R}$ & SR 5-year/10-year: 29\%/20\% median survival time 27 months \\
\hline Ogata (2005) & 76 & $\mathrm{R}$ & DFS 5-year cumul. 27\%; lung DFS 5-year cumul. 35\%; Cumul. SR 5-year 32\% (after first resection) \\
\hline $\begin{array}{l}\text { Kanemitsu } \\
\quad(2010)\end{array}$ & & $\mathrm{P}$ & SR 1-year/2-year/3-year/5-year: 96.6\%/84.5\%/70.5\%/48.9\% \\
\hline Park (2010) & 195 & $\mathrm{R}$ & SR 5-year: $71.2 \%$ \\
\hline $\begin{array}{l}\text { Headrick } \\
\quad(2001)\end{array}$ & 58 & $\mathrm{R}$ & SR 5-year/10-year: 30\%/16\% \\
\hline $\begin{array}{r}\text { Hornbech } \\
\quad(2011)\end{array}$ & 53 & $\mathrm{R}$ & $\begin{array}{l}\text { Estimated SR 5-year: 50.3\%, median } 33.5 \text { months (total group); 0\%, median } 15.4 \text { months (also hepatic } \mathrm{M}+\text { ); } \\
61.7 \% \text {, median } 28.6 \text { months (no-hepatic } \mathrm{M}+\text { ) }\end{array}$ \\
\hline Inoue (2004) & 128 & $\mathrm{R}$ & Estimated SR 5-year: $45.3 \%$ \\
\hline Chen (2009) & 84 & $\mathrm{R}$ & DFS 5-year/10-year: 34.4\%/30.6\%; SR 5-year/10-year: 60.5\%/48.4\% \\
\hline Hwang (2010) & 125 & $\mathrm{P}$ & Median 37 months \\
\hline Riquet (2010) & 117 & $\mathrm{R}$ & SR 5-year/10-year: 41\%/27\%, median 45 months \\
\hline Rama (2008) & 62 & $\mathrm{R}$ & DFS mean $52 \pm 6$ months; SR 3-year/5-year/10-year: $61 \% / 48 \% / 11 \%$, mean $67 \pm 16$ months \\
\hline $\begin{array}{r}\text { Takakura } \\
\text { (2009) }\end{array}$ & 56 & $\mathrm{R}$ & DFS 13 months (range, 2-35 months); cumul. SR 3-year/5-year 64.9\%/48.2\% \\
\hline Lin (2009) & 63 & $\mathrm{P}$ & DFS 5-year: 19.5\%; SR 5-year/10-year: 43.9\%/19.5\% \\
\hline $\begin{array}{l}\text { Zabaleta } \\
\quad(2011)\end{array}$ & 84 & $\mathrm{R}$ & SR 3-year/5-year: $70.2 \% / 54.3 \%$, median 72 months (range, 0-129 months) \\
\hline $\begin{array}{l}\text { Pfannschmidt } \\
\quad(2003)\end{array}$ & 167 & $\mathrm{R}$ & Cumul. SR 5-year: $32.4 \%$, median 40.2 months \\
\hline $\begin{array}{l}\text { Higashiyama } \\
\text { (2003) }\end{array}$ & 100 & $\mathrm{R}$ & SR 3-year/5-year: $62.2 \% / 49.4 \%$ \\
\hline $\begin{array}{r}\text { Nakajima } \\
(2007)\end{array}$ & 143 & $\mathrm{R}$ & SR 5-year: 49.3\% (open resection group), 39.5\% (thoracoscopy group) \\
\hline Shiono (2004) & 87 & $\mathrm{R}$ & SR 5-year: $61.4 \%$ \\
\hline $\begin{array}{r}\text { Sakamoto } \\
\quad(2010)\end{array}$ & 59 & $\mathrm{R}$ & SR 5-year: 69.9\% (CRC patients) \\
\hline $\begin{array}{l}\text { Kanzaki } \\
\text { (2011) }\end{array}$ & 156 & $\mathrm{R}$ & SR 3-year/5-year/10-year: 71.4\%/56.2\%/44\% \\
\hline Rolle (2006) & 91 & $\mathrm{R}$ & SR 5-year: 41\% (complete resection); 7\% (incomplete resection) \\
\hline Rolle (2006) & 91 & $\mathrm{R}$ & $\begin{array}{l}\text { SR: } 1 \text {-year/3-year/5-year: 81\%/53\%/35\% (all } 328 \text { patients) 85\%/59\%/41\% (complete resection) 60\%/23\%/7\% } \\
\text { (incomplete resection) }\end{array}$ \\
\hline
\end{tabular}


For a summary of the survival rates provided in the articles, see Table 3. For analysis 2- and 5-year survival rates were chosen, since these were the two most reported survival data. In papers which did not provide either of these, but did have a Kaplan-Meier curve, 2- and 5-year survival rates were extracted manually from this curve.

For RFA 2-year survival rate (SR) ranged from $64-73 \%{ }^{12-15}$ and 5-year SR from $34.9-45 \%{ }^{12,15}$ The surgical series provided 2 -year SR range from $64-88 \% .{ }^{17-25,28-32}$ The 5-year SR reported for surgery ranged from $29-71.2 \%{ }^{17-25,27-35}$ As seen in various uni- and multivariate analyses ${ }^{12-14,35}$, survival outcomes are influenced by the size of the intrapulmonary lesion, especially in patients treated with RFA. Tumors larger than $3 \mathrm{~cm}$ give significantly shorter longterm survival and local control rates than lesions smaller than $3 \mathrm{~cm}$.

Mean and median tumor size were difficult to compare between surgery and RFA studies, due to differences in reporting, but seemed similar for both therapies (Table 2).

\section{Complications}

In only 20 articles treatment-related mortality and/or morbidity were described, which are tabulated (Table 4). The remaining 7 studies were not included in this table.

Treatment-related mortality rates were reported by three of the studies investigating RFA. ${ }^{13-15}$ Mortality rate was $0 \%$ in all three, but was defined differently (Table 4). Morbidity and complication rates differed but all four RFA studies reported pneumothorax as the most common post-treatment complication ( $22 \%$ of sessions ${ }^{12}$, $29 \%$ of patients ${ }^{13,14}$ and $45 \%$ of patients ${ }^{15}$ ).
Three surgical studies reported treatment-related mortalities (Zabaleta et al. ${ }^{27}$, Pfannschmidt et al. ${ }^{17}$, Nakajima et al. ${ }^{36}$ ) of $2.4 \%$, $1.8 \%$ and $1.4 \%$, respectively. All other fourteen studies which reported complications showed mortality rates of $0 \%{ }^{13-15,18-}$ $20,24,26,28,31-35,37,38$

Morbidity rates ranged from $0-14.5 \%$, with large variations in the reporting of complications. Some studies for example reported only major morbidity (e.g. Kanzaki et $a .^{32}$ ) whereas others reported all complications that occurred.

\section{Discussion}

Since the proposal of the existence of oligo-metastases by Hellmann et al., ${ }^{3}$ local therapies have been used for treating these few distant metastases with curative intent. For oligo-metastases in the lung, the most commonly used local treatment modalities are surgery, RFA and SBRT, see Fig. 2. To our knowledge the present study was the first to systematically review and try to compare metastasectomy, RFA and SBRT for patients with pulmonary metastases of colorectal cancer with regard to local tumor control, OS and complications.

By systematically searching databases, we identified 27 published studies, of which four were dealing with RFA and 23 with surgery. ${ }^{12-38}$ No eligible studies were found on SBRT, even after adjusting the selection criteria to a minimum of 25 patients and a minimal follow-up of 12 months. For this reason, no analysis was made on SBRT data. Of the included four RFA series, three studies were from the same institute, which has to be kept in mind given the possibility that some patient data may be used in multiple studies and therefore may bias outcome data. In two surgical

Table 4

Complications.

\begin{tabular}{|c|c|c|c|}
\hline Study & $\begin{array}{l}\text { Mortality } \\
\text { rate }\end{array}$ & $\begin{array}{l}\text { Morbidity } \\
\text { rate }\end{array}$ & Complications \\
\hline $\begin{array}{c}\text { Yamakado } \\
\text { (2009) }\end{array}$ & $\mathrm{N} / \mathrm{A}$ & $\mathrm{N} / \mathrm{A}$ & $\begin{array}{l}\text { Pneumothorax in 31/140 sessions (22.1\%), chest tube placement in } 18 \text { sessions; aseptic pleuritis with chest tube placement } \\
\text { in } 2 \text { sessions (1.4\%); Minor complication } 13 / 140 \text { (9.3\%); Major complication } 20 / 140(14.3 \%)\end{array}$ \\
\hline Yan (2006) & $0 \%$ & $42 \%$ & $\begin{array}{l}\text { Post-RFA: pneumothorax (16), fever (6), pleural effusion ( } 4 \text { ), pleuritic chest pain ( } 2 \text { ); chest drain } n=10 \text {, nine for } \\
\text { pneumothorax, } 1 \text { for pleural effusion. Duration of chest drain } 2 \pm 2 \text { days }\end{array}$ \\
\hline Yan (2007) & $0 \%$ & $42 \%$ & Intrapulmonary bleeding (5); pneumothorax (16); pleural effusion (4); persistent pleuritic chest pain more than 1 week (2) \\
\hline Chua (2010) & $0 \%$ & $\mathrm{~N} / \mathrm{A}$ & $\begin{array}{l}\text { Morbidity: pneumothorax (66), pleural effusion (16), consolidation (10), bleeding (1), pleuritic chest pain (12); chest tube } \\
\text { placement ( } 45 \text { ) of which one ptn developed severe consolidation and lung abcess with empyema }\end{array}$ \\
\hline $\begin{array}{l}\text { Brouquet } \\
\quad(2011)\end{array}$ & $0 \%$ & $4 \%$ & For lung resection only: Mortality $0 \%$ (Treatment-related); Morbidity 4\% ( $n=4)$ (postoperative) \\
\hline Koga (2006) & $0 \%$ & $\mathrm{~N} / \mathrm{A}$ & $\mathrm{N} / \mathrm{A}$ \\
\hline Ogata (2005) & $0 \%$ & $1 \%$ & Hemorrhage $n=1$ \\
\hline Park (2010) & $0 \%$ & $\mathrm{~N} / \mathrm{A}$ & N/A \\
\hline $\begin{array}{l}\text { Headrick } \\
\quad(2001)\end{array}$ & $0 \%$ & $12 \%$ & Ileus (2), bile leak (1), chyle leak (1), incisional hernia (1), postoperative bleeding (1), pulmonary embolus (1) \\
\hline Hwang (2010) & $0 \%$ & $\mathrm{~N} / \mathrm{A}$ & $\mathrm{N} / \mathrm{A}$ \\
\hline Riquet (2010) & $0 \%$ & $14.5 \%$ & $\begin{array}{l}\text { Atrial fibrillation (2); persistent air leaks (6); atelectasis (1); empyema (2); hemorrhage (2); pneumonia (1); recurrent } \\
\text { nerve palsy (1); miscellaneous (2) }\end{array}$ \\
\hline Rama (2008) & $0 \%$ & $8 \%$ & Bronchopleural fistulae (2); acute renal insufficiency (1), hemorrhage (1); wound infection (1) \\
\hline $\begin{array}{l}\text { Zabaleta } \\
\quad(2011)\end{array}$ & $\begin{array}{l}2.4 \% \\
(n=2)\end{array}$ & $8.3 \%$ & $\begin{array}{l}\text { Persistent air leaks (3); nosocomial pneuomonia (1); partial intestinal obstruction (1); atelectasis (1) requiring } \\
\text { bronchoscopy, haematothorax (1) (requiring reintervention) }\end{array}$ \\
\hline $\begin{array}{l}\text { Pfannschmidt } \\
\text { (2003) }\end{array}$ & $\begin{array}{l}1.8 \% \\
(n=3)\end{array}$ & $\mathrm{N} / \mathrm{A}$ & Postoperative deaths by septic multiorgan failure after pneumonia (2); death by sudden cardiac failure (1) \\
\hline $\begin{array}{r}\text { Nakajima } \\
\text { (2007) }\end{array}$ & $1.4 \%$ & $\mathrm{~N} / \mathrm{A}$ & Postoperative deaths (pulmonary thromboembolism; gastrointestinal bleeding) (2) \\
\hline Shiono (2004) & $0 \%$ & N/A & $\mathrm{N} / \mathrm{A}$ \\
\hline $\begin{array}{r}\text { Sakamoto } \\
(2010)\end{array}$ & $0 \%$ & $9.8 \%$ & $\begin{array}{l}\text { Persistent air leak ( } 3 \text { ); pneumonia (2); cerebral infarction, postoperative bleeding, atelectasis, duodenal ulcer, chylothorax, } \\
\text { ileus, urinary tract infection, empyema ( } 1 \text { each) }\end{array}$ \\
\hline $\begin{array}{l}\text { Kanzaki } \\
\quad(2011)\end{array}$ & $0 \%$ & $0 \%$ & $\mathrm{~N} / \mathrm{A}$ \\
\hline Rolle (2006) & $0 \%$ & $\mathrm{~N} / \mathrm{A}$ & Major morbidity: persistent air leaks (2); intrapleural bleeding (2); late pneumothorax (2) \\
\hline Rolle (2006) & $0 \%$ & N/A & Major morbidity: persistent air leaks (2); intrapleural bleeding (2); late pneumothorax (2) \\
\hline
\end{tabular}

Perioperative; In-hospital; Treatment-related; Operative; Postoperative; 30-day.

Including hepatic surgery complications.

Metastasis group. 

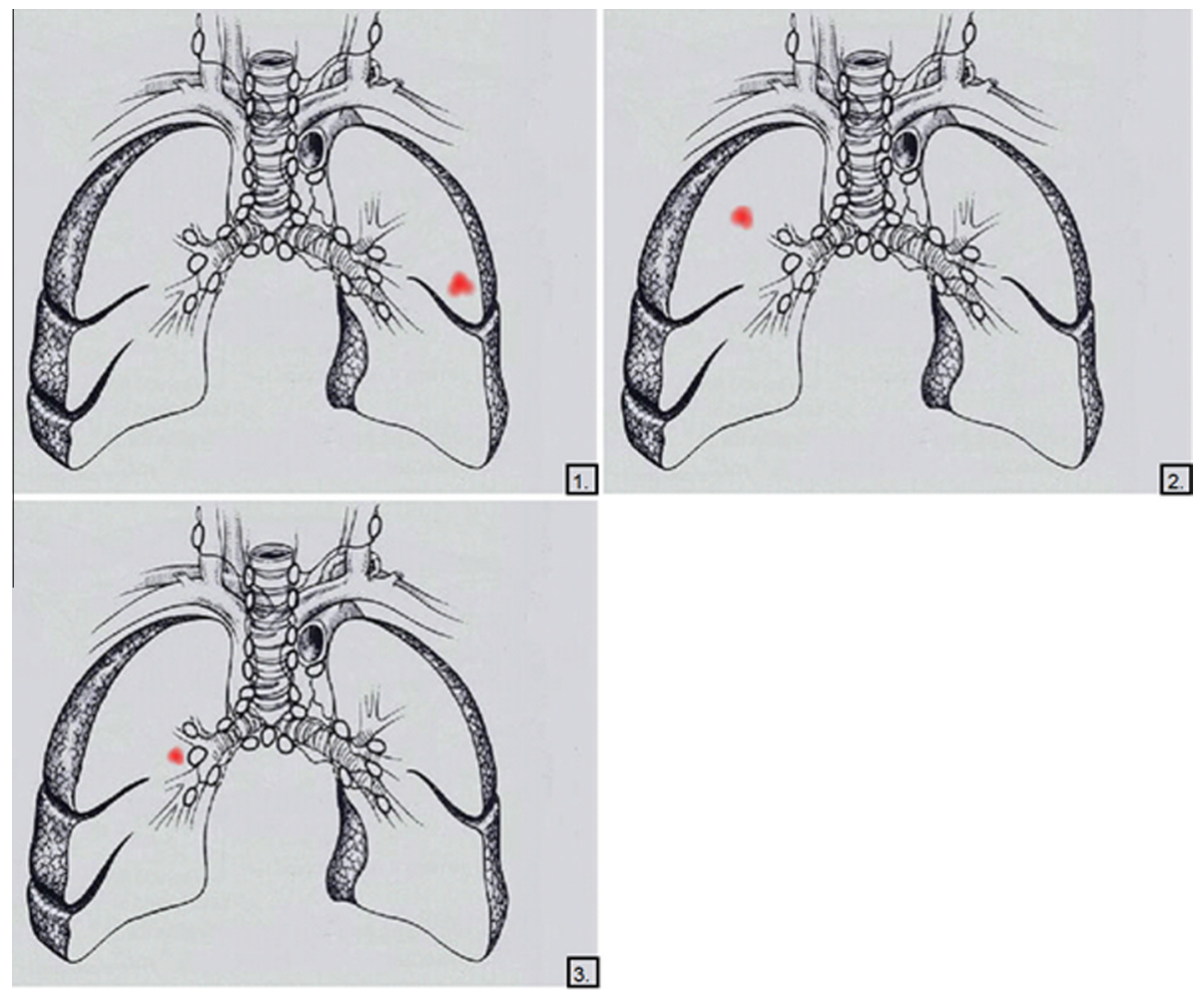

1. Peripheral zone (1): Surgery $\rightarrow$ RFA/ SBRT

2. Middle zone (2): RFA/ Surgery/SBRT

3. Central zone (3): SBRT $\longrightarrow$ RFA/ Surgery

Fig. 2. Leuven-Maastricht guideline map for the local treatment of lung metastases.

series, the same patient population is used, which was the reason to use these data only once in our analysis.

In the identified papers, outcome data were reported in various ways, with different parameters mentioned or sometimes incomplete data. This resulted in difficulties analyzing and comparing these outcomes. Also, diagnostic differences may have influenced outcome, such as differences in CT slice thickness. This is not always reported clearly but could influence outcome results. Survival outcomes used for analysis are either reported in the papers or extracted manually from Kaplan-Meier curves and weighted for number of patients afterwards. Since there is a strong heterogeneity in the reporting of outcome data, the quality op comparisons between surgery and RFA are difficult en should be approached with care. Comparing RFA and surgery, the differences in OS are small and in the absence of randomized trial, no firm conclusions can be drawn. Since tumors larger than $3 \mathrm{~cm}$ give significantly shorter long-term survival and local control rates than lesions smaller than $3 \mathrm{~cm}$, differences in tumor size in the included patients in the RFA and surgery studies may have influenced the results.

Investigating complication rates, RFA seems to have better outcomes, since the mortality rate in 3 studies was $0 \%$, compared to mortality rates of $1.4-2.4 \%$, reported in three surgical studies. ${ }^{17,27,32}$ However, morbidity rates are higher in the RFA studies compared with the surgery papers, with an average of $45.5 \%$ for the three studies ${ }^{12-15}$ compared with $6.6 \%$ in nine surgical studies $^{18-20,27,28,32,34,35,37}$ (Table 4). The problem however, is that probably not all complications are reported, mainly in these surgery articles, in which sometimes only major complications are reported.
Because of the wide variety in data, caused by patient selection and the lack of phase III trials, no conclusions can be drawn on effectiveness of surgery for lung metastases of colorectal cancer in comparison to RFA.

This review points out that there is a need for prospective, randomized studies, including studies that investigate the effectiveness and long-term survival of SBRT and RFA. It is to our opinion very likely that SBRT represents an effective treatment method in this population. Based on outcomes from studies investigating SBRT for primary lung cancer, e.g. Timmerman et al. ${ }^{39}$, Senan et al. ${ }^{40}$ and a review by Fernando et al. ${ }^{9}$ in which SBRT and RFA seemed to be effective and useful treatment modalities for high-risk primary lung cancer patients, we assume that outcomes for pulmonary metastases may be comparable to those for primary lung cancer. This assumption is confirmed by the prospective, multicentre study of Lencioni et al. $^{41}$ in which both NSCLC and pulmonary metastasized colorectal cancer patients were treated with RFA. This paper was not included in this review because of a followup of only 15 months. However, results show that there is no difference in response between these two patient-groups. Overall survival at 1 and 2 years were 70\% and 48\% respectively for NSCLC and $89 \%$ and $66 \%$ for patients with colorectal metastases.

\section{Conclusions}

Because of the lack of randomized trials, no firm conclusions can be drawn about the relative merits of surgery, RFA and SBRT for colorectal lung metastases. However, only surgical series provide large prospective series with large patient numbers. 
Therefore, surgery is the treatment of choice in this condition. Other therapy options such as RFA and SBRT have been reported to be successful as well, but most series are at present too small, retrospective and or have a follow-up that is too short to draw definitive conclusions. Nevertheless, retrospective RFA studies support its effectiveness and this modality may thus be regarded as a less defined alternative for surgery. Finally, because of our stringent a priori inclusion criteria for published series, we could not include radiotherapy studies in this review. Indeed, published studies of SBRT for colo-rectal metastases have still a short followup, which is not surprising in view of the relative new technology that SBRT is and its only recent wide-spread use. Assuming an analogous efficacy for colo-rectal metastases as for primary NSCLC, SBRT series when more maturated may well establish radiotherapy as the third local treatment option for patients with colo-rectal lung metastases. Ideally, randomised trials should be performed to define the value of all three modalities, taking into account patient and disease characteristics.

\section{Conflict of interest}

None declared.

\section{References}

1. Jemal A, Bray F, Center MM, Ferlay J, Ward E, Forman D. Global cancer statistics. CA Cancer J Clin 2011;61:69-90.

2. IKNL. Comprehensive cancer centre NL Website. p. Retrieved December 12th 2011, Available from: http://iknl.nl.

3. Hellman S, Weichselbaum RR. Oligometastases. J Clin Oncol 1995;13:8-10.

4. Giacchetti S, Itzhaki M, Gruia G, Adam R, Zidani R, Kunstlinger F, et al. Longterm survival of patients with unresectable colorectal cancer liver metastases following infusional chemotherapy with 5-fluorouracil, leucovorin, oxaliplatin and surgery. Ann Oncol 1999;10:663-9.

5. Rusthoven KE, Kavanagh BD, Burri SH, Chen C, Cardenes H, Chidel MA, et al. Multi-institutional phase I/II trial of stereotactic body radiation therapy for lung metastases. J Clin Oncol 2009;27:1579-84.

6. Treasure T, Fallowfield L, Lees B, Farewell V. Pulmonary metastasectomy in colorectal cancer: the PulMiCC trial. Thorax 2012;67:185-7.

7. Fiorentino F, Hunt I, Teoh K, Treasure T, Utley M. Pulmonary metastasectomy in colorectal cancer: a systematic review and quantitative synthesis. $J R$ Soc Med 2010;103:60-6.

8. Siva S, MacManus M, Ball D. Stereotactic radiotherapy for pulmonary oligometastases: a systematic review. J Thorac Oncol 2010;5:1091-9.

9. Fernando HC, Schuchert M, Landreneau R, Daly BT. Approaching the high-risk patient: sublobar resection, stereotactic body radiation therapy, or radiofrequency ablation. Ann Thorac Surg 2010;89:S2123-7.

10. Akobeng AK. Principles of evidence based medicine. Arch Dis Child 2005;90:837-40.

11. da Costa Santos CM, de Mattos Pimenta CA, Nobre MR. The PICO strategy for the research question construction and evidence search. Rev Lat Am Enfermagem 2007; 15:508-11.

12. Yamakado K, Inoue Y, Takao M, Takaki H, Nakatsuka A, Uraki J, et al. Long-term results of radiofrequency ablation in colorectal lung metastases: single center experience. Oncol Rep 2009;22:885-91.

13. Yan TD, King J, Sjarif A, Glenn D, Steinke K, Morris DL. Percutaneous radiofrequency ablation of pulmonary metastases from colorectal carcinoma: prognostic determinants for survival. Ann Surg Oncol 2006;13:1529-37.

14. Yan TD, King J, Sjarif A, Glenn D, Steinke K, Al-Kindy A, et al. Treatment failure after percutaneous radiofrequency ablation for nonsurgical candidates with pulmonary metastases from colorectal carcinoma. Ann Surg Oncol 2007; 14:1718-26.

15. Chua TC, Sarkar A, Saxena A, Glenn D, Zhao J, Morris DL. Long-term outcome of image-guided percutaneous radiofrequency ablation of lung metastases: an open-labeled prospective trial of 148 patients. Ann Oncol 2010;21:2017-22.

16. Kanemitsu Y, Kato $T$, Komori $K$, Fukui $T$, Mitsudomi $T$. Validation of nomogram for predicting overall survival after resection of pulmonary metastases from colorectal cancer at a single center. World J Surg 2010;34:2973-8.

17. Pfannschmidt J, Muley T, Hoffmann H, Dienemann H. Prognostic factors and survival after complete resection of pulmonary metastases from colorectal carcinoma: experiences in 167 patients. J Thorac Cardiovasc Surg 2003;126:732-9.

18. Brouquet A, Vauthey JN, Contreras CM, Walsh GL, Vaporciyan AA, Swisher SG, et al. Improved survival after resection of liver and lung colorectal metastases compared with liver-only metastases: a study of 112 patients with limited lung metastatic disease. J Am Coll Surg 2011;213:62-9.

19. Rama N, Monteiro A, Bernardo JE, Eugenio L, Antunes MJ. Lung metastases from colorectal cancer: surgical resection and prognostic factors. Eur J Cardiothorac Surg 2009;35:444-9.

20. Ogata Y, Matono K, Hayashi A, Takamor S, Miwa K, Sasatomi T, et al. Repeat pulmonary resection for isolated recurrent lung metastases yields results comparable to those after first pulmonary resection in colorectal cancer. World J Surg 2005;29:363-8.

21. Chen FS, Hanaoka N, Sato K, Fujinaga T, Sonobe M, Shoji T, et al. Prognostic factors of pulmonary metastasectomy for colorectal carcinomas. World J Surg 2009;33:505-11.

22. Lin BR, Chang TC, Lee YC, Lee PH, Chang KJ, Liang JT. Pulmonary resection for colorectal cancer metastases: duration between cancer onset and lung metastasis as an important prognostic factor. Ann Surg Oncol 2009;16: 1026-32.

23. Takakura Y, Miyata Y, Okajima M, Okada M, Ohdan H. Short disease-free interval is a significant risk factor for intrapulmonary recurrence after resection of pulmonary metastases in colorectal cancer. Colorectal Dis 2010;12:e68-75.

24. Koga R, Yamamoto J, Saiura A, Yamaguchi T, Hata E, Sakamoto M. Surgical resection of pulmonary metastases from colorectal cancer: four favourable prognostic factors. Jpn J Clin Oncol 2006;36:643-8.

25. Hornbech K, Ravn J, Steinbruchel DA. Outcome after pulmonary metastasectomy analysis of 5 years consecutive surgical resections 20022006. J Thorac Oncol 2011;6:1733-40.

26. Hwang MR, Park JW, Kim DY, Chang HJ, Kim SY, Choi HS, et al. Early intrapulmonary recurrence after pulmonary metastasectomy related to colorectal cancer. Ann Thorac Surg 2010;90:398-405.

27. Zabaleta J, Aguinagalde B, Fuentes MG, Bazterargui N, Izquierdo JM, Hernandez CJ, et al. Survival after lung metastasectomy for colorectal cancer: importance of previous liver metastasis as a prognostic factor. Eur J Surg Oncol 2011;37:786-90.

28. Headrick JR, Miller DL, Nagorney DM, Allen MS, Deschamps C, Trastek VF, et al. Surgical treatment of hepatic and pulmonary metastases from colon cancer. Ann Thorac Surg 2001;71:975-9.

29. Inoue $M$, Ohta $M$, Iuchi $K$, Matsumura $A$, Ideguchi $K$, Yasumitsu T, et al. Benefits of surgery for patients with pulmonary metastases from colorectal carcinoma. Ann Thorac Surg 2004;78:238-44.

30. Higashiyama M, Kodama K, Higaki N, Takami K, Murata K, Kameyama M, et al. Surgery for pulmonary metastases from colorectal cancer: the importance of prethoracotomy serum carcinoembryonic antigen as an indicator of prognosis. Jpn J Thorac Cardiovasc Surg 2003;51:289-96.

31. Shiono S, Ishii G, Nagai $K$, Yoshida J, Nishimura M, Murata $Y$, et al. Histopathologic prognostic factors in resected colorectal lung metastases. Ann Thorac Surg 2005;79:278-83.

32. Kanzaki R, Higashiyama M, Oda K, Fujiwara A, Tokunaga T, Maeda J, et al. Outcome of surgical resection for recurrent pulmonary metastasis from colorectal carcinoma. Am J Surg 2011;202:419-26.

33. Park JS, Kim HK, Choi YS, Kim K, Shim YM, Jo J, et al. Outcomes after repeated resection for recurrent pulmonary metastases from colorectal cancer. Ann Oncol 2010;21:1285-9.

34. Riquet M, Foucault C, Cazes A, Mitry E, Dujon A, Barthes FL, et al. Pulmonary resection for metastases of colorectal adenocarcinoma. Ann Thorac Surg 2010;89:375-80.

35. Sakamoto M, Murakawa T, Kitano K, Murayama T, Tsuchiya T, Nakajima J. Resection of solitary pulmonary lesion is beneficial to patients with a history of malignancy. Ann Thorac Surg 2010;90:1766-72.

36. Nakajima J, Murakawa T, Fukami T, Takamoto S. Is thoracoscopic surgery justified to treat pulmonary metastasis from colorectal cancer? Interact Cardiovasc Thorac Surg 2008;7:212-6. discussion 6-7.

37. Rolle A, Pereszlenyi A, Koch R, Bis B, Baier B. Laser resection technique and results of multiple lung metastasectomies using a new $1318 \mathrm{~nm} \mathrm{Nd}$ :YAG laser system. Lasers Surg Med 2006;38:26-32.

38. Rolle A, Pereszlenyi A, Koch R, Richard M, Baier B. Is surgery for multiple lung metastases reasonable? A total of 328 consecutive patients with multiple-laser metastasectomies with a new 1318-nm Nd:YAG laser. J Thorac Cardiovasc Surg 2006;131:1236-42.

39. Timmerman R, Heinzerling J, Abdulrahman R, Choy H, Meyer JL. Stereotactic body radiation therapy for thoracic cancers: recommendations for patient selection, setup and therapy. Front Radiat Ther Oncol 2011;43:395-411.

40. Senan S, Palma DA, Lagerwaard FJ. Stereotactic ablative radiotherapy for stage I NSCLC: recent advances and controversies. J Thorac Dis 2011;3:189-96.

41. Lencioni R, Crocetti L, Cioni R, Suh R, Glenn D, Regge D, et al. Response to radiofrequency ablation of pulmonary tumours: a prospective, intention-totreat, multicentre clinical trial (the RAPTURE study). Lancet Oncol 2008;9:621-8. 